\title{
EL BUEN USO DE LAS TIC EN LOS PLANES DOCENTES DE ENSEÑANZA JURÍDICA: REFLEXIÓN EN TORNO A LA PROTECCIÓN DE LOS DERECHOS PERSONALÍSIMOS DEL ALUMNO
}

\section{THE GOOD USE OF TIC IN THE TEACHING PLANS OF LEGAL EDUCATION: CONSIDERATION ON THE PROTECTION OF THE PERSONAL RIGHTS OF THE STUDENT}

http://dx.doi.org/10.15304/ie.28.5210

\author{
Ma Nieves Alonso García \\ Universidad Isabel I \\ marianieves.alonso@uil.es
}

\author{
David Carrizo Aguado \\ Universidad de León \\ dcara@unileon.es
}

\section{RESUMEN}

En los últimos años los avances tecnológicos han abierto la posibilidad de que la práctica y planificación docente se ejercite a través de herramientas digitales. Las TIC se convierten en un instrumento encaminado a fortalecer la relación entre profesor/alumno y a facilitar la participación, deliberación y colaboración entre los mismos. Este sistema de enseñanza tecnocrático entra en colisión con el derecho a la protección de datos de carácter personal, constitucionalmente reconocido y cuyo amparo se ha visto reforzado con la entrada en vigor del Reglamento (UE) 2016/679 del Parlamento Europeo y del Consejo de 27 de abril de 2016, relativo a la protección de las personas físicas en lo que respecta al tratamiento de datos personales y a la libre circulación de estos datos.

Palabras Clave: TIC, práctica y planificación docente, Google Calendar, protección de datos.

\begin{abstract}
In recent years, technological advances have opened up the possibility of teaching practice and planning being exercised through digital tools. ICT become an instrument aimed at strengthening the relationship between teacher and student and facilitate participation, deliberation and collaboration between them. This technocratic education system collides with the right to the protection of constitutionally recognized personal data whose protection has been reinforced by the entry into force of Regulation (EU) 2016/679 of the European Parliament and of the Council of 27 April 2016 on the protection of natural persons with regard to the processing of personal data and on the free movement of such data. Keywords: ICT, teaching practice and planning, Google Calendar, data protection.
\end{abstract}




\section{LA REPERCUSIÓN DE LAS TIC EN LA PRAXIS DOCENTE DEL DERECHO}

\subsection{Justificación disciplinar}

La coyuntura presente reclama de juristas, filósofos y teóricos del derecho una conciencia tecnológica, es decir, una actitud reflexiva, crítica y responsable antes los nuevos problemas que en las diversas esferas del acontecer social suscita la tecnología y ante los que ni el derecho, ni quienes lo aplican o estudian pueden permanecer insensibles (Pérez Luño, 2001, p. 45).

Esta reflexión de Pérez Luño constituye la esencia de esta contribución desde la perspectiva docente en las asignaturas de Derecho Constitucional y Derecho internacional privado. En los planes de estudio de todo Estado social y democrático de Derecho se incorpora como materia el Derecho Constitucional en tanto en cuanto, la Constitución como cúspide del ordenamiento jurídico tiene como objetivo velar por la existencia de una sociedad organizada sobre una base democrática y participativa (Balaguer Callejón, 2016). Por su naturaleza, es el basamento sobre el que descansan el resto de las ramas del Derecho.

Sobre este sustrato, el Derecho internacional privado parte de otro aspecto incuestionable de la realidad social: las relaciones humanas tienden a superar las fronteras político-jurídicas de un país y por tanto queden conectadas con más de un sistema jurídico. Los movimientos migratorios, la nueva sociedad digital, la globalización en la economía, la apertura de los mercados... han resultado foco vislumbraste en la actividad de los particulares hacia sus relaciones, vinculándose, por tanto, con uno o varios ordenamientos jurídicos fuera de su esfera normativa y que, trasciende del ámbito de aplicación de uno solo de los ordenamientos (López Tarruella, 2017). Consecuentemente, la pluralidad de sistemas jurídicos determina la importancia objetiva y funcional del Derecho internacional privado. Realmente, cada Estado posee su propio ordenamiento jurídico el cual debe convivir con normas de carácter regional y supraestatal. Es por ello que, una misma conducta o actuación pueda estar conectada con más de un ordenamiento o incluso con más de una jurisdicción. Esta idea, muestra la contraposición a una situación privada interna que se caracteriza porque todos sus elementos están vinculados a un único Estado o sistema jurídico. Es menester del Derecho internacional privado asegurar respuestas adecuadas a las relaciones jurídicas privadasinternacionales procurando facilitar las relaciones tanto personales, así como, comerciales entre los diversos sujetos implicados. (Calvo Caravaca y Carrascosa González, 2017; Esplugues, Iglesias Buhigues y Palao, 2017; Fernández Rozas y Sánchez Lorenzo, 2016; Michinel, 2011).

Desde esta disciplina, se contribuye al conocimiento de la tutela judicial efectiva y el ejercicio de los derechos, los problemas vinculados al estatuto internacional de las personas y la determinación del Derecho aplicable a las situaciones y relaciones jurídicas de tráfico externo ${ }^{1}$. Para velar por el

Sobre el contenido del Derecho internacional privado se ha discutido mucho; ahora bien, los tres sectores fundamentales sobre el que se asienta la disciplina son: en primer término, la determinación de los tribunales competentes para conocer de un posible litigio; en segundo lugar, qué ley se debe aplicar para conocer el fondo del asunto y, por último, qué eficacia puede llegar a tener en otros Estados un pronunciamiento judicial (Garcimartín, 2017). 
cumplimiento eficaz de estas competencias, es precisa su conexión con el Derecho Constitucional a través del cual se fomenta el aprendizaje de los valores democráticos propios de una sociedad civil democrática y se garantiza el conocimiento escrupuloso de los derechos fundamentales y principios que deben velar en el tráfico jurídico internacional y en el reconocimiento de los derechos de las personas extranjeras en nuestro país (Cruz Villalón, 1992; Rodríguez Ruiz, 2015).

\subsection{Contextualización social y su imbricación con el ámbito educativo}

En el ámbito político, y trasladable al ámbito educativo, en tanto en cuanto refleja elementos que puede ser referenciales, el estudio del INJUVE sobre jóvenes, política y $\mathrm{TIC}^{2}$ acredita que la falta de confianza de la población entre 18 y 30 años hacia canales de participación tradicionales no es incompatible con el interés de la política. La necesidad de implicar a los jóvenes en el debate a través del uso de las TIC en el proceso docente puede convertirse en una herramienta eficaz para el aprendizaje.

Este estudio, extrapolado a la docencia, evidencia que las nuevas tecnologías pueden suponer una motivación y un medio para potenciar un feedback entre profesores y alumnos (Barrios Espinosa y García Mata, 2008; Hamodi Galán, 2013; Lozano Martínez y Tamez Vargas, 2017; Medel Marchena y Jaén Martínez, 2016; Santana Bonilla, Jiménez Jiménez, Pintor Díaz y Hernández Rivero, 2017). Ambos sujetos deben prepararse para coexistir con la tecnología de forma armoniosa.

El objetivo que persigue esta contribución es establecer un equilibrio armonioso entre el fugaz desarrollo de las herramientas tecnológicas con la protección y tutela de los derechos fundamentales del colectivo de los alumnos.

Los docentes son el principal sujeto al que va dirigido, a fin de clarificar y aportar una visión jurídica al ejercicio de su actividad, fundamentalmente en aquellas directamente relacionadas con el tratamiento e datos de carácter personal.

\subsection{Aplicación de las nuevas tecnologías en la docencia jurídica}

La innovación docente en la era de la información está intrínsecamente unida al dominio de las TIC (Pérez de la Fuente, 2013). Desde este prisma, las reflexiones sobre la innovación educativa tecnológica se centran en considerar que esta supone una transformación significativa. Asimismo, implica un cambio en la concepción de la enseñanza, que repercute en la práctica educativa y que tiene una finalidad: la mejora de la calidad del aprendizaje. A este respecto, la innovación no es un fin, sino un medio para mejorar la calidad y conseguir los objetivos que se proponen los centros educativos. De este modo, no supone necesariamente una creación, pero sí un cambio que conlleva mejoras en la calidad de la enseñanza basadas en una intervención deliberada (De Pablos Pons y Jiménez Cortés, 2007).

2 Disponible en: http://www.injuve.es/observatorio/infotecnologia/jovenes-y-nuevas-tecnologias (Consulta: 15-5-2018). 
En los últimos años los avances tecnológicos han abierto la posibilidad de que la práctica docente se ejercite a través de la introducción de los MOOC (Massive Open Online Courses) ${ }^{3}$, el empleo de herramientas online como "Moodle", la incorporación de medios y redes sociales en la docencia o la implementación de sistemas de evaluación alternativos.

Las vías por las que circulará esta participación virtual de los alumnos serán los foros de debate virtuales, los blogs, las webs institucionales, las consultas online, o incluso las redes sociales, fundamentalmente Twitter (Abad Arenas, 2015). La potenciación de estas nuevas vías puede suponer significativos cambios en las relaciones entre docentes y alumnos.

Las TIC se convierten en un instrumento encaminado a fortalecer el interés de los alumnos por las tareas docentes que, pueden experimentar mejoras a través de estas tecnologías que facilitan su intervención, el acceso a la información y las relaciones de colaboración. Su inclusión en el proceso de enseñanza debe promover la creatividad, la expresión personal y contribuir a la formación de una sociedad civil informada, participativa, crítica y seria. La Universidad debe ser capaz de integrar el desarrollo tecnológico para hacer más fácil e inmediata la consecución de sus objetivos.

La principal paradoja es la aspiración a que este entorno digital sea capaz de suplir una realidad física que inste a prescindir o minimizar el papel del docente. Valorar los aspectos positivos y negativos de estos recursos tecnológicos se entiende solo si la tecnología es una ayuda, un complemento o un refuerzo del sistema educativo que contribuya a fortalecer el aprendizaje. El reto es convertir a las TIC en herramientas útiles para la creación de entornos diferentes para el aprendizaje y la comunicación entre los participantes en la acción formativa. Para el cumplimiento de este fin se requiere una participación activa de todos los agentes para evitar que estas herramientas se conviertan solo en el vehículo para la distribución de mensajes.

Una percepción inicial se puede sintetizar en la prolija situación que deriva de la irrupción de las nuevas tecnologías en el ámbito educativo. Así, si las TIC contribuyen a visibilizar las principales dificultades de compresión de los alumnos, su aportación-al fomento del diálogo y del debate tiene el riesgo de estimular la polarización de los participantes.

3 La metodología docente para los MOOC debe contar con los siguientes elementos: "Vídeo Lecciones: Exposiciones magistrales del tutor grabadas en pequeños clips de vídeo de como máximo 15 minutos de duración, lo que constituye el método principal para comunicar los contenidos durante el curso y suelen tener una periodicidad semanal. Localizadas en la red y accesibles desde cualquier dispositivo pueden ser seguidas por los alumnos a su propio ritmo y no de manera secuencial; Cuestionarios. Son pruebas en línea que permiten al alumno demostrar su conocimiento sobre los temas que se está capacitando; Tareas. Ejercicios que están disponibles durante la semana y que han de completar de manera correcta, pues de su resolución dependerá la solvencia en el examen posterior; Exámenes. Son las calificaciones globales después de responder con mayor o menor fiabilidad las preguntas que sobre los conceptos expuestos durante la semana se han tratado en las tareas y cuestionarios. Los exámenes tratarán los mismos temas que en las evaluaciones anteriores, pero de una manera más profusa; Foros y/o Tablones. Cuestiones que se plantean a los profesores de manera directa. Estas preguntas pueden ser visualizadas por el resto de los alumnos y votar por ellas. Los instructores responderán a las preguntas más votadas" (Caldera-Serrano y León-Moreno, 2015, p. 302). 
En esta misma línea, su contribución a la estimulación del interés de los estudiantes puede verse lastrado por la desidia que desemboque en participaciones erráticas, esporádicas o carentes de compromiso. Sin duda, a la rapidez de sus flujo informativos y de sus inmediatas respuestas le surge la sombra de la ausencia de reflexión sobre las que se cimentan. Igualmente, al fortalecimiento del control hay que añadir la debilidad del anonimato de quien lo ejerce, la irresponsabilidad que le acompaña y las dudas sobre la neutralidad de los buscadores y de la información desde la que se formulan los posicionamientos. En suma, a los aspectos positivos se le pueden enfrentar sombras que exigen cautela y mesura en ese reto digital que es inevitable (Noguera, 2013).

\subsection{La planificación docente con la herramienta Google Calendar}

El uso de la herramienta Google Calendar (Abad Ruiz, 2017) ha sido decisivo en la planificación horaria del curso académico. La diversidad de asignaturas, la estructuración en distintos grupos, junto con la tipología de créditos A, B1, B3, conlleva una tarea ardua que se ha facilitado mediante el uso de esta aplicación colaborativa de Google.

A través de la misma, se han configurado diariamente el tramo horario de cada una de las disciplinas a fin de que el alumno, desde sus dispositivos electrónicos, pueda conocer la hora de inicio y fin de las asignaturas, el aula en la que se imparte la materia y el tipo de crédito. La sincronización de esta aplicación con un teléfono móvil o tablet permitirá un conocimiento directo e instantáneo del alumno con su programación diaria.

En su elaboración se procedió a clasificar las asignaturas con diversos colores. La propia aplicación permite elegir entre once tonos distintos asignando un matiz predeterminado para cada una de ellas. Mediante la fijación de las fechas de inicio y final del curso académico, descontando los períodos no lectivos y considerando la carga horaria correspondiente a cada una de las asignaturas impartidas en el Grado, se elabora un calendario académico proporcionadamente delimitado dentro de los planes de estudio.

El mismo proceso se ha llevado a cabo para la determinación de las fechas, horas y aulas en las que se realizarán los exámenes en las convocatorias ordinaria y extraordinaria. De igual modo, el alumno podrá acceder desde sus dispositivos al calendario de exámenes. Esta herramienta facilita la distribución de las fechas de los exámenes al hacer más visible el período temporal transcurrido desde la finalización de las clases teórico-prácticas y la realización de los mismos. Ese impasse es una de las principales preocupaciones manifestadas por los alumnos, que podrá ser solventada con mayor facilidad a través de esta aplicación.

Asimismo, el profesorado responsable de cada asignatura utilizará este instrumento tecnológico para la programación en detalle de la asignatura, de modo que el alumno con carácter previo al inicio de cada clase conozca el contenido de la guía docente que será impartido un determinado día. La finalidad perseguida con este sistema de planificación es facilitar una interrelación entre docente y alumno que otorgue un mayor dinamismo y un seguimiento más riguroso y coherente de la materia tratada. 


\section{EL REGLAMENTO EUROPEO DE PROTECCIÓN DE DATOS: LOS SISTEMAS DE EVALUACIÓN Y EL TRATAMIENTO DE DATOS PERSONALES DEL ALUMNO}

\subsection{Observación inicial}

El establecimiento de un sistema que permita evaluar el aprendizaje continuo del estudiante es el ámbito donde el diseño de este sistema de enseñanza tecnocrático entra en colisión con el derecho a la protección de datos de carácter personal.

La evaluación desde una perspectiva tradicional se ha concebido como una actividad final encargada de cerrar el ciclo de formación de los estudiantes y, por tanto, al margen de la actividad de aprendizaje. En cambio, en la actualidad, la evaluación, de acuerdo con las modernas teorías pedagógicas, se entiende como un proceso integrado e interrelacionado con ciclos de formación desde el momento inicial de planificación hasta la comprobación de los resultados en el cual resulta de gran utilidad el uso de plataformas docentes virtuales (Oliver Cuello, 2010, p. 79).

En este contexto, la entrada en vigor del Reglamento (UE) 2016/679 del Parlamento Europeo $y$ del Consejo de 27 de abril de 2016, relativo a la protección de las personas físicas en lo que respecta al tratamiento de datos personales y a la libre circulación de estos datos ${ }^{4}$ ha aportado luz antes los nuevos interrogantes que plantea el tratamiento de datos en el ámbito digital y los derechos de los usuarios.

Dicha norma europea ha llevado a cabo la urgente tarea, que se veía reclamando a nivel comunitario, para adecuar y armonizar de manera uniforme en los Estados miembros el nivel de protección de los derechos y libertades de las personas físicas por lo que se refiere al tratamiento lícito, leal y transparente de dichos datos (Díaz Díaz, 2016; Plaza Penadés, 2017).

En él se refleja la idea de que el tratamiento de datos personales debe estar concebido para servir a la humanidad, que el derecho a la protección de datos no es un derecho absoluto, sino que debe considerarse en relación con su función en la sociedad, lo que exige en su regulación mantener el equilibrio con otros derechos fundamentales de acuerdo al principio de proporcionalidad.

El Reglamento, como norma jurídica de Derecho comunitario, posee alcance general y eficacia directa en todos los Estados miembros de la Unión, de modo que no obliga a su trasposición al Derecho interno de cada Estado. De ahí, que los particulares lo puedan invocar a efectos de hacer efectiva la tutela jurisdiccional ante los tribunales nacionales o comunitarios desde el 25 de mayo de 2018 tal y como prevé su artículo 99.2.

\subsection{Los principios de transparencia y de limitación del plazo de conservación}

Vinculado con el ámbito educativo, es especialmente relevante la regulación relativa a la legitimación para el tratamiento de datos y el deber de informar. La Ley Orgánica 15/1999, de 13 de diciembre, de Protección de Datos de Carácter Personal ${ }^{5}$ establece como obligaciones vinculadas

DOUE núm. 119, de 4 de mayo de 2016.

BOE núm. 298, de 14 de diciembre de 1999. 
a la información que se ha de facilitar a las personas interesadas en el momento en que se soliciten los datos, la existencia del fichero o tratamiento, su finalidad y destinatario, el carácter obligatorio o no de la respuesta, así como de sus consecuencias, la posibilidad de ejercitar los derechos de acceso, rectificación, cancelación y oposición y la identidad y datos de contacto del responsable del tratamiento (Guasch y Soler, 2015).

Con la entrada en vigor del Reglamento Europeo de Protección de Datos se establecen como requisitos adicionales en cuanto a la necesidad de informar a las personas interesadas, incorporando en líneas generales, los siguientes detalles: los datos de contacto del delegado de Protección de Datos, en su caso, la base jurídica o legitimación para el tratamiento, el plazo o los criterios de conservación de la información, la existencia de decisiones automatizadas o elaboración de perfiles, la previsión de transferencias a terceros países y el derecho a presentar una reclamación antes las Autoridades de Control. Asimismo, y en el caso de que los datos no se obtengan del propio interesado se prevé como requisito adicional el origen y las categorías de los mismos (Grande Sanz, 2016; Ortiz López, 2017).

En la protección de los datos personales de los alumnos priman dos de los principios recogidos en el citado Reglamento: el principio de transparencia (Garrós, 2018) y el principio de limitación del plazo de conservación. Desde el primero, se vela porque el tratamiento de datos de carácter personal se realice de manera lícita, leal y transparente. Es decir, el derecho a estar informado de manera clara e inequívoca sobre el tratamiento que tiene el sujeto titular de los datos. Conforme al mismo, se impone al responsable del fichero la obligación de facilitar la información que debe dar el sujeto titular tanto con carácter previo como ex post si en un futuro se destinan a una finalidad diferente por la cual se recabaron sus datos personales. Desde el segundo, se prevé que los datos sean conservados de tal manera que se permita la identificación de los interesados, únicamente, el tiempo que sea necesario para los fines del tratamiento. Como excepción al plazo de conservación, los datos personales podrán ser mantenidos durante un tiempo superior siempre y cuando sean utilizados, exclusivamente, con fines de archivo de interés público, de investigación científica, histórica o estadística.

A este respecto, los exámenes y/o pruebas de evaluación, así como los trabajos del alumnado, se conservarán hasta la finalización del curso siguiente, excepto aquellos que den lugar a revisión y recurso que se conservarán hasta que finalice el último de los recursos posibles. Los modelos de examen, por el contrario, serán de conservación permanente.

\subsection{Análisis crítico de la evaluación: la publicación de las notas}

La publicación de las calificaciones de las pruebas evaluativas constituye un supuesto de cesión o comunicación de datos. Conforme al artículo 3 i) de la Ley Orgánica de Protección de Datos, se entiende por cesión de datos "toda revelación de datos realizada a persona distinta del afectado". El término "revelación" implica que se integra en este concepto todos aquellos actos en los que la información se muestre a terceros, con independencia de que los mismos obtengan de modo efectivo o no aquélla. 
Sobre este particular, la Ley Orgánica 4/2007, de 12 de abril, por la que se modifica la Ley Orgánica 6/2001, de 21 de diciembre, de Universidades ${ }^{6}$, en su Disposición adicional vigésimo primera relativa a la protección de datos de carácter personal, establece la obligación de las universidades de adoptar las medidas de índole técnica y organizativa necesarias que garanticen la seguridad de los datos de carácter personal y eviten su alteración, tratamiento o acceso no autorizados. En esta misma línea, prevé que no será preciso el consentimiento de los estudiantes para la publicación de los resultados de las pruebas relacionadas con la evaluación de sus conocimientos y competencias ni de los actos que resulten necesarios para la adecuada realización y seguimiento de dicha evaluación. Asimismo, no será preciso el consentimiento del personal de las universidades para la publicación de los resultados de los procesos de evaluación de su actividad docente, investigadora y de gestión realizados por la universidad o por las agencias o instituciones públicas de evaluación.

Posee especial relevancia en la normativa europea la introducción de la figura del Delegado de protección de datos (Martínez Moriel, 2017; Recio Gayo, 2016; Rojo Fernández-Matinot, 2018). El Reglamento (UE) 2016/679 establece su obligatoriedad para autoridades y organismos públicos, responsables o encargados que tengan entre sus actividades principales las operaciones de tratamiento que requieran una observación habitual y sistemática de interesados a gran escala y responsables o encargados que tengan entre sus actividades principales el tratamiento a gran escala de datos sensibles.

Los dos últimos supuestos, la Ley Orgánica de Protección de Datos los circunscribe a los colegios profesionales y sus consejos generales, los centros docentes y las Universidades públicas y privadas, las entidades que exploten redes y presten servicios de comunicaciones electrónicas, los prestadores de servicios de la sociedad de la información cuando elaboren a gran escala perfiles de los usuarios del servicio, las entidades de ordenación, supervisión y solvencia de entidades de crédito; los establecimientos financieros de crédito, las entidades aseguradoras y reaseguradoras y de servicios de inversión, los distribuidores y comercializadores de energía eléctrica y de gas natural.

A tenor de la normativa expuesta, su presencia tendrá carácter obligatorio en el ámbito de las Universidades tanto españolas como europeas. Sus funciones girarán en torno a la obligación de informar y asesorar sobre protección de datos a la organización, evaluar el nivel de riesgo e impacto en la privacidad, notificar a los sujetos sobre violaciones de datos y cooperar con las autoridades de supervisión (Stanzione, 2016).

\section{A MODO DE CONCLUSIÓN}

Las herramientas tecnológicas son la clave para potenciar nuevas capacidades de aprendizaje, en particular la capacidad de comunicación profesor/alumno y en la misión pedagógica, instructiva y evaluadora.

6 BOE núm. 89, de 13 de abril de 2007. 
El futurible ya es presente y la nueva etapa de la enseñanza universitaria es el resultado de la combinación de un contexto sociopolítico, dentro de la sociedad de la información, con el impacto de los avances tecnológicos sobre las instituciones educativas. Sus características se desgranan en torno a la accesibilidad, la responsabilidad y la eficacia.

La materia prima de la sociedad tecnológica es la transmisión de información. La formación de dicha información es el quid. La masiva información disponible para los alumnos es un elemento que refuerza el aprendizaje. El docente tiene que adoptar un rol activo en la transmisión de conocimientos a los alumnos. Para ello el recurso a las nuevas tecnologías es clave. Es importante que en esa estrategia de divulgación de contenidos se recupere un referente, el valor de la especialización del docente en los contenidos compartidos.

Es interesante abogar por la existencia de clases magistrales en las que el docente asuma el reto de contribuir a impulsar el interés de los alumnos en los contenidos impartidos a la par que facilitar la realización de actividades y su evaluación a través de las herramientas virtuales. Una calidad de la formación transmitida vinculada a un entorno tecnocrático desde el que se divulgue no puede asimilarse a vulgarizar.

Todos estos cambios requieren de un equipo técnico que vele por la protección de los datos personales. La política debe inspirar los cambios, el derecho debe ofrecer sus garantías y los técnicos físicos y digitales deben asegurar la operatividad de cada propuesta que ha de beneficiar el interés de los docentes y alumnos.

\section{REFERENCIAS BIBLIOGRÁFICAS}

Abad Arenas, E. (2015). Las distintas modalidades tutoriales impartidas mediante la utilización de las TIC en la UNED: las funciones del profesor-tutor ante este nuevo marco docente y discente. En A. M. Delgado García e I. Beltrán de Heredia Ruiz, Las TIC y las redes sociales y la docencia del derecho. Barcelona: Huygens.

Abad Ruiz, F. (2017). Implementación de una agenda escolar colaborativa con Google Calendar. Proyecto y tutorial. e-CO: Revista digital de educación y formación del profesorado, 14, pp.131-165.

Balaguer Callejón, F. (2016). El derecho constitucional. En F. Balaguer Callejón (Coord.), Manual de derecho constitucional. Madrid: Tecnos.

Barrios Espinosa, E. y García Mata, J. (2008). Suministro de feedback a ensayos sobre diarios en un programa de prácticas de enseñanza: propuesta de una modalidad apoyada en tic y estudio de percepciones del alumnado. Pixel-Bit: Revista de medios y educación, 33, pp. 73-84.

Caldera-Serrano, J. y León-Moreno, J. A. (2015). MOOC (Massive Online Open Courses) como método-plataforma educativa en el ámbito universitario. Documentación de las ciencias de la información, 38, pp. 301-310. DOI: https://doi.org/10.5209/rev_DCIN.2015.v38.50821

Calvo Caravaca, A-L. y Carrascosa González, J. (2017). Derecho Internacional Privado, Vol. I, $17^{\mathrm{a}}$ ed. Granada: Comares. 
Cruz Villalón, P. (1992). Dos cuestiones de titularidad de derechos: los extranjeros, las personas jurídicas. Revista española de derecho constitucional, 35, pp. 63-84.

De Pablos Pons, J. y Jiménez Cortés, R. (2007). Buenas prácticas con TIC apoyadas en las Políticas Educativas: claves conceptuales y derivaciones para la formación en competencias ECTS. Revista Latinoamericana de Tecnología Educativa, 2, pp. 15-28.

Díaz Díaz, E. (2016). El nuevo Reglamento General de Protección de Datos de la Unión Europea y sus consecuencias jurídicas para las instituciones. Revista Aranzadi Doctrinal, 6, pp. 155-190.

Esplugues, C.; Iglesias Buhigues, J. L. y Palao, G. (2017). Derecho Internacional Privado, $11^{\mathrm{a}}$ ed. Valencia: Tirant lo Blanch.

Fernández Rozas, J. C. y Sánchez Lorenzo, S. A. (2016). Derecho Internacional Privado, 9a ed. Navarra: Civitas Thomson Reuters.

Garcimartín, F. J. (2017). Derecho Internacional Privado. $4^{\mathrm{a}}$ ed., Navarra: Civitas Thomson Reuters.

Garrós, I. (2018). El principio de transparencia y el derecho a la protección de datos personales: Comentarios a propósito del Reglamento sobre protección de las personas físicas en lo que respecta al tratamiento de datos personales y a la libre circulación de estos datos. Actualidad Administrativa, 2, versión on-line.

Grande Sanz, M. (2016). La transferencia internacional de datos personales: presente y futuro. Diario La Ley, 8808, versión on-line.

Guasch, V. y Soler, J. R. (2015). El interés legítimo en la protección de datos. RDUNED, Revista de derecho UNED, 16, pp. 417-438. DOI: https://doi.org/10.5944/rduned.16.2015.15245

Hamodi Galán, C. (2013). El feedback entre profesorado y alumnado como elemento potente para la regulación de los errores. En V. M. López Pastor; R. Monjas Aguado y C. Vallés Rapp (Coords.), La Evaluación de Competencias en Educación Superior. La evaluación formativa y compartida en enseñanza universitaria, Madrid: Red Nacional de Evaluación Formativa.

López Tarruella, A (2017). Manual de Derecho Internacional Privado, $2^{\circ}$ ed. Alicante: ECU.

Lozano Martínez, F. y Tamez Vargas, L. A. (2014). Retroalimentación formativa para estudiantes de educación a distancia. RIED: revista iberoamericana de educación a distancia, 2, pp. 197221. DOI: https://doi.org/10.5944/ried.17.2.12684

Martínez Moriel, I. (2017). El delegado de protección de datos, como garante de la privacidad en las organizaciones. Actualidad jurídica Aranzadi, 930, versión on-line.

Medel Marchena, I. y Jaén Martínez, A. (2016). La Gamificación en el proceso de mediación del aprendizaje en Enseñanza Superior: Análisis comparativo de aplicaciones de tipo feedback. En R. Roig-Vila (Coord.), Tecnología, innovación e investigación en los procesos de enseñanza-aprendizaje. Barcelona: Octaedro.

Michinel, M. A. (2011). El Derecho Internacional Privado en los tiempos hipermodernos. Madrid: Dykinson.

Noguera, A. (2013). Reflexionando sobre las TIC: ventajas y peligros de las TIC en la docencia del derecho. En A. Cerrillo i Martínez y A. M. Delgado García (Coords.), Buenas prácticas docentes en el uso de las TIC en el ámbito del derecho. Barcelona: Huygens.

Oliver Cuello, R. (2010). La evaluación del aprendizaje y la plataforma docente Moodle. En A. Cerrillo i Martínez y A. M. Delgado García (Coords.), Docencia del Derecho y tecnologías de la información y la comunicación. Barcelona: Huygens. 
Ortiz López, P. (2017). Regulando la privacidad del futuro. Análisis de la Propuesta de Reglamento europeo de e-Privacy y su interconexión con el Reglamento General de Protección de Datos. Diario La Ley, 10, Sección Ciberderecho, versión on-line.

Pérez de la Fuente, O. (2013). Una experiencia de innovación docente con nuevas tecnologías para aplicación del modelo Bolonia desde la Filosofía del derecho. Revista de Educación y derecho, 9, versión-on-line.

Pérez Luño, E. A. (2001). Ensayos de informática jurídica. México: Fontamara.

Plaza Penadés, J. (2017). Implementando el nuevo Reglamento General europeo de Protección de datos. Revista Aranzadi de Derecho y nuevas tecnologías, 43, pp. 19-21.

Recio Gayo, M. (2016). El delegado de la protección de datos. En J. L. Piñar Mañas (Dir.), Reglamento general de protección de datos: hacia un nuevo modelo europeo de privacidad. Barcelona: Reus.

Rodríguez Ruiz, B. (2015). Participación y ciudadanía más allá del sufragio. Los derechos de participación de las personas extranjeras. Revista de estudios políticos, 169, pp. 45-74.

Rojo Fernández-Matinot, I. (2018). El Delegado de Protección de Datos. Diario La Ley, 9189, versión on-line.

Santana Bonilla, P.; Jiménez Jiménez, F.; Pintor Díaz, P. y Hernández Rivero, V. M. (2017). Cómo implicar al alumnado en la evaluación formativa. El valor del feedback. En Á. Vega Navarro y D. Stendardi (Coords.), Imaginar y Comprender la Innovación en la Universidad: VII Jornadas de Innovación Educativa de la Universidad de la Laguna. La Laguna: Universidad de La Laguna, Servicio de Publicaciones.

Stanzione, M. G. (2016). Il regolamento europeo sulla privacy: origini e ambito di applicazione. Europa e diritto privato, 4, pp. 1249-1264. 